\title{
Human case of Onchocerca lupi infection, Germany, August 2014
}

A Bergua ${ }^{1,2,3}$, B Hohberger ${ }^{1,2,3}$, J Held $^{2,4}$, B Muntau ${ }^{5}$, E Tannich5, D Tappe (tappe@bnitm.de)5

1. Department of Ophthalmology and Eye Clinic, Friedrich Alexander Universität Erlangen-Nürnberg (FAU), Erlangen, Germany

2. Universitätsklinikum Erlangen, Erlangen, Germany

3. Both authors contributed equally to this study

4. Mikrobiologisches Institut, Friedrich Alexander Universität Erlangen-Nürnberg (FAU), Erlangen, Germany

5. Bernhard Nocht Institute, Hamburg, Germany

Citation style for this article:

Bergua A, Hohberger B, Held J, Muntau B, Tannich E, Tappe D. Human case of Onchocerca lupi infection, Germany, August 2014. Euro Surveill.

2015;20(16):pii=21099. Available online: http://www.eurosurveillance.org/ViewArticle.aspx?Articleld=21099

Onchocerca lupi, a nematode parasite infecting dogs and cats with a hitherto unknown arthropod vector, is also being recognised as a parasite also responsible for human eye infections. Here we describe a case of human eye infection diagnosed molecularly by nematode $12 \mathrm{~S}$ rDNA PCR in a German patient who had travelled to Tunisia and Turkey. The patient recovered after treatment with antibiotic and anti-inflammatory therapy.

In this report we describe an eye infestation with Onchocerca lupi diagnosed in August 2014 by PCR in a German patient who had previously travelled to Tunisia and Turkey; the patient did not report a history of trauma or insect bite in the ocular or periocular region. $O$. lupi is an emerging nematode parasite found in dogs and cats in southern Europe (Portugal and Greece), central Europe (Germany and Hungary), and the United States (US) [1].

\section{Case description}

In August 2014, a 28-year-old German patient presented at the Department of Ophthalmology and Eye Clinic, University Hospital Erlangen-Nürnberg with a painful localised swelling of the bulbar conjunctiva supratemporally of the right eye, accompanied by severe episcleral and conjunctival hyperaemia. The swelling, mimicking a nodular scleritis, had developed two months before. It subsequently increased in size, became painful, and did not change under local and systemic steroids. The symptoms were not accompanied by fever or local lymphadenopathy. The patient did not report any history of trauma or insect bite in the ocular or periocular region, nor was animal contact reported. The patient had travelled to Tunisia (in June 2013, in the city of Sousse and its surroundings along the Mediterranean Sea) and Turkey (in July 2012, in the city of Alanya and its surroundings along the Mediterranean Sea). Visual acuity, intraocular pressure, anterior chamber, vitreous body, and the fundus of both eyes were normal. Inflammatory parameters

\section{FIGURE 1}

Macroscopic image of the Onchocerca lupi nematode removed from the eye of a German patient, August 2014

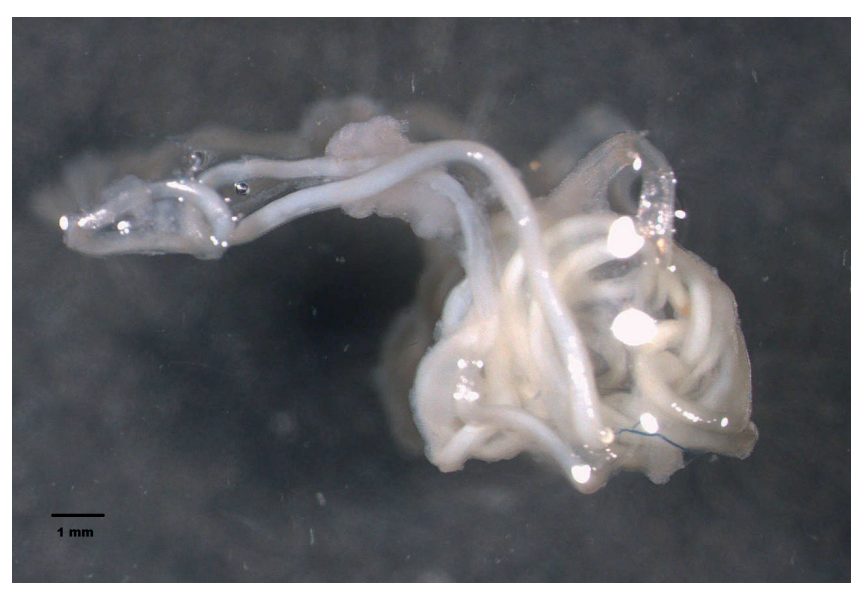

Recovered full subconjunctival specimen. Magnification x 15 .

and differential blood count were normal, but IgE was elevated $(167 \mathrm{U} / \mathrm{mL}$; norm: $<100)$. Slit lamp examination revealed a subconjunctival mass that after careful excision appeared to be an inactive nematode (Figure 1).

\section{Investigation of the cause of infection}

The cuticle of the parasite showed external ridges resembling the cuticular serration of Dirofilaria repens, and ocular dirofilariosis was suspected. A nematodespecific PCR targeting the $12 \mathrm{~S}$ rDNA [2] was conducted after DNA extraction from the removed parasite. The $393 \mathrm{bp}$ amplicon was sequenced. BLAST analysis (www. ncbi.nlm.hih.gov/blast) showed $100 \%$ nt identity with O. Lupi (GenBank accession number $\mathrm{GU}_{3} 65879$, canine infection in Portugal) and a $99 \%$ sequence identity with 0 . lupi from human infections in Iran and Turkey (JN863696 and HQ207645, respectively). The sequence 
Molecular phylogeny of various Onchocerca species and other filaria species pathogenic for humans based on partial sequences of parasite $12 \mathrm{~S}$ rRNA genes

C. elegans_JF896455

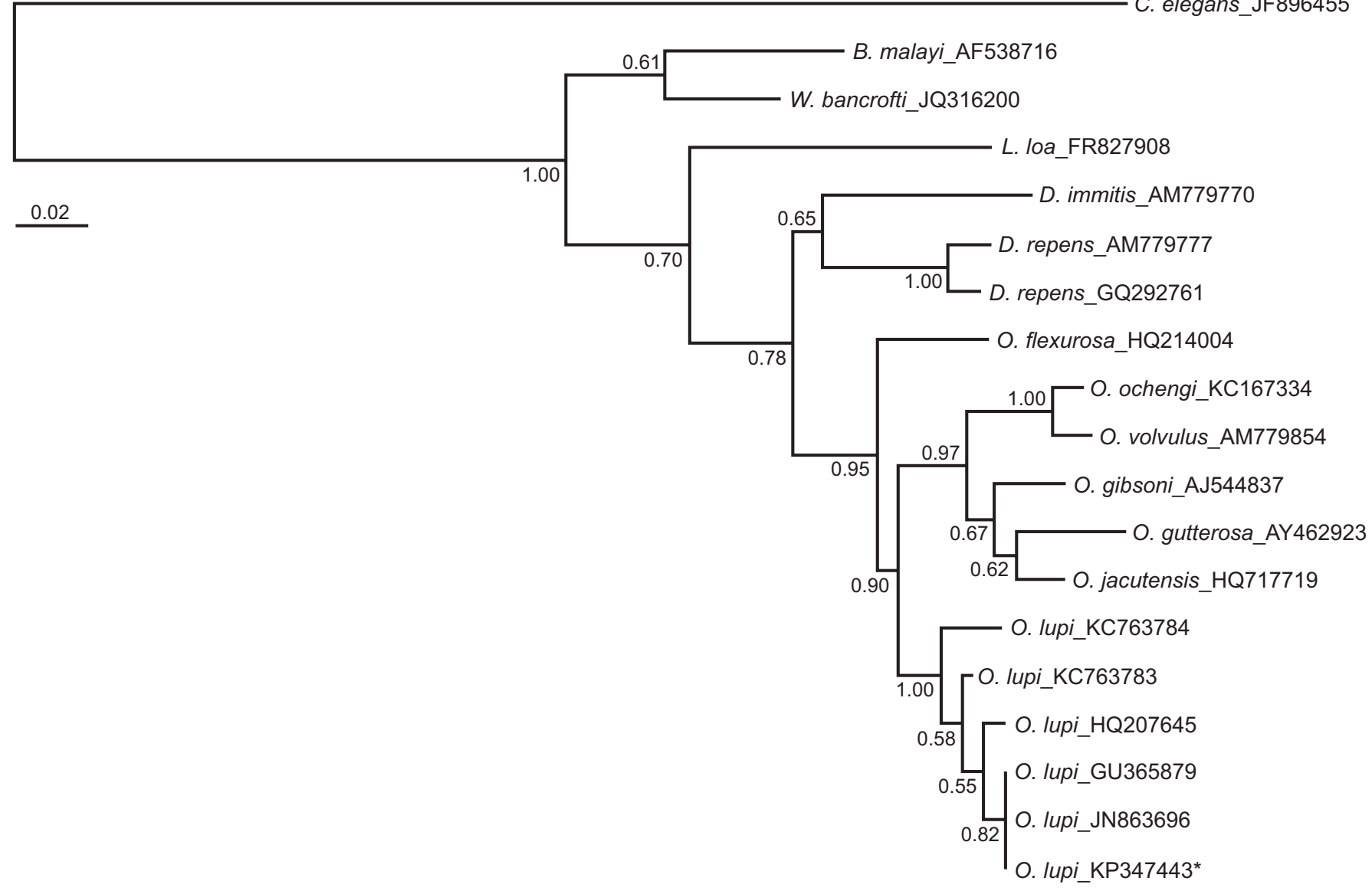

The phylogenetic sequence tree was inferred using a Bayesian approach with 1,000,00o generations assuming the HKY model, and samples were taken every 10oth generation. Bayesian posterior probabilities are given at the nodes and the scale bar is equal to 0.2 expected substitutions per site. GenBank accession numbers of the sequences used are provided and Caenorhabditis elegans was chosen as outgroup. The sequence of the specimen recovered from the patient's eye (KP347443) is marked with asterisk. It clusters in particular with Onchocerca lupi sequences from Portugal (canine infection, GU365879 (100\% nt identity, 393 of 393 nt)) and Iran (human infection, JN863696 (99\% nt identity, 393 of 395 nt)), followed by those from Turkey (human infection, HQ207645 (99\% nt identity, 373 of 375 nt)) and California (canine infections, KC763783 (100\% nt identity, 326 of $326 \mathrm{nt})$ and KC 763784 (97\% nt identity, 383 of $396 \mathrm{nt})$ ).

from the patient's isolate was deposited in GenBank (KP347443) and clustered with European, Middle East, and Californian $O$. lupi sequences from humans and animals (Figure 2).

\section{Follow-up of the patient}

One month after this episode, a second, but smaller subconjunctival swelling developed supranasally on the same side. The mass impressed the retina, as observed by funduscopy and echography. Again, there was no lymphadenopathy and differential blood count was normal. Surgical exploration and subsequent histological examination revealed no obvious nematode structures; however, PCR was again positive for 0 . lupi. This discrepancy might be explained by the fact that the PCR was performed from a different, not microscopically examined part of the excised tissue than the part which underwent histopathological examination. Both lesions healed well under antibiotic therapy with topic tobramycin and cefuroxime in order to prevent bacterial superinfection, and anti-inflammatory therapy. Ivermectin or albendazole were not administered as complete resection was assumed.

\section{Discussion}

O. lupi is a nematode parasite infecting dogs and cats with a hitherto unknown arthropod vector, usually found in southern and central Europe and in the US; it is also being recognised as a parasite responsible for human eye infections. In dogs, nodular eye lesions with gravid females and production of microfilariae may develop [1]. The zoonotic significance of $O$. lupi was first highlighted in 2002 in two suspected human cases from Albania and Russia [3] that had occurred close to regions where the parasite had been described in a wolf and dogs [4]. Only recently, further and definitive human infections have been described from 2011 onwards in Turkey [5-7], Iran [8], Tunisia [7], and the US 
$[9,10]$. The clinical picture in humans is characterised by the development of a bulbar subconjunctival nodule $[5-7,10]$. In one case also multiple eye nodules were observed [8]. An extradural infection with spinal cord compression was seen in a 22-month-old girl [9]. The presumed arthropod vector for 0 . lupi is unknown, but might be a simuliid black fly in analogy to the biology of Onchocerca volvulus [11], the agent of human river blindness. Besides more infections reported in dogs $[1,4]$, several human eye infections with 0 . lupi have been reported. Symptoms in humans and dogs are strikingly similar, involving the eye and its appendices. As demonstrated by our case and a previously reported one [8], also multiple 0 . lupi nodules can occur during human infection - either in parallel [8] or, as seen in our case, successively. In the patient described here, molecular methods unequivocally identified $O$. lupi as the causative agent. The investigation of skin snips, as taken for the detection of microfilariae in 0 . volvulus infections and in canine $O$. lupi disease, has only been reported in one human case with an $O$. lupi infection [9]. In that case, caused by a gravid female 0 . Iupi containing microfilariae, skin snips were negative. In all eight clearly diagnosed human cases we found in the literature, surgical excision led to a complete cure. Only in two cases $[9,10]$ additional anthelminthic drugs (ivermectin and/or albendazole) were administered. In one case dexamethasone was applied after surgery and cryotherapy because of generalised urticaria [8]. The incubation time of the disease is unknown. In one report, a 'fly-bite' on the eye had been described 30 days before manifestation of conjunctivitis and 58 days before pain and swelling [5].

So far, in contrast to canine disease $[1,4,12]$, definitive human 0 . lupi infections have not been described in Europe except for two cases living in Istanbul, Turkey $[5,7]$. The nematode's $12 \mathrm{~S}$ rDNA sequence here was identical to a sequence from 0 . lupi in dogs from Portugal, and showed $99 \%$ similarities to sequences derived from human cases in Turkey and Iran (2 nt difference each in a stretch of $375 \mathrm{nt}$ and of $395 \mathrm{nt}$, respectively). The parasite had been detected in a local dog from an animal shelter in Germany in 2002 [12], however, the source of the dog's infection remained unclear. In theory, similar to a recent report on the first autochthonous case of human dirofilariosis in Germany soon after the detection of $D$. repens in German dogs [13], the human 0 . lupi infection reported here could have also been acquired autochthonously in Germany - by a hitherto unknown vector, even though this does not seem very likely. Tunisia and Turkey, countries with reported human infections [5-7] were visited by our patient 11 and 22 months before symptom onset, respectively, and are more likely to have been the places of infection. Due to increasing global travel activities, international migration, and importation of dogs, more cases of human 0 . lupi infections might be diagnosed in the future.
Acknowledgments

We are grateful to Prof. Christian Bogdan, University Clinic of Erlangen, for critical reading of the manuscript.

Conflict of interest

None declared.

Authors' contributions

Patient examination and surgery: $A B, B H$. Molecular and morphological analyses: JH, DT, ET, BM. Wrote the manuscript: $A B, B H, J H, B M, E T, D T$.

References

1. Otranto D, Dantas-Torres F, Giannelli A, Latrofa MS Papadopoulos E, Cardoso L, et al. Zoonotic Onchocerca lupi infection in dogs, Greece and Portugal, 2011-2012. Emerg Infect Dis. 2013;19(12):2000-3. http://dx.doi.org/10.3201/ eid1912.130264 PMID:24274145

2. Casiraghi M, Bain O, Guerrero R, Martin C, Pocacqua V, Gardner SL, et al. Mapping the presence of Wolbachia pipientis on the phylogeny of filarial nematodes: evidence for symbiont loss during evolution. Int J Parasitol. 2004;34(2):191-203. http://dx.doi.org/10.1016/j.ijpara.2003.10.004 PMID:15037105

3. Sréter T, Széll Z, Egyed Z, Varga I. Subconjunctival zoonotic onchocerciasis in man: aberrant infection with Onchocerca lupi? Ann Trop Med Parasitol. 2002;96(5):497-502. http:// dx.doi.org/10.1179/000349802125001267 PMID:12194710

4. Sréter T, Széll Z. Onchocercosis: a newly recognized disease in dogs. Vet Parasitol. 2008;151(1):1-13. http://dx.doi. org/10.1016/j.vetpar.2007.09.008 PMID:17951007

5. Otranto D, Sakru N, Testini G, Gürlü VP, Yakar K, Lia RP, et al. Case report: First evidence of human zoonotic infection by Onchocerca lupi (Spirurida, Onchocercidae). Am J Trop Med Hyg. 2011;84(1):55-8. http://dx.doi.org/10.4269/ ajtmh.2011.10-0465 PMID:21212202

6. Ilhan HD, Yaman A, Morishima Y, Sugiyama H, Muto M, Yamasaki $\mathrm{H}$, et al. Onchocerca lupi infection in Turkey: a unique case of a rare human parasite. Acta Parasitol. 2013;58(3):384-8. http://dx.doi.org/10.2478/s11686-013-01528 PMID:23990437

7. Otranto D, Dantas-Torres F, Cebeci Z, Yeniad B, Buyukbabani $\mathrm{N}$, Boral OB, et al. Human ocular filariasis: further evidence on the zoonotic role of Onchocerca lupi. Parasit Vectors. 2012;5(1):84. http://dx.doi.org/10.1186/1756-3305-5-84 PMID:22541132

8. Mowlavi G, Farzbod F, Kheirkhah A, Mobedi I, Bowman DD, Naddaf SR. Human ocular onchocerciasis caused by Onchocerca lupi (Spirurida, Onchocercidae) in Iran. I Helminthol. 2014;88(2):250-5. http://dx.doi.org/10.1017/ So022149X13000060 PMID:23388686

9. Eberhard ML, Ostovar GA, Chundu K, Hobohm D, Feiz-Erfan I, Mathison BA, et al. Zoonotic Onchocerca lupi infection in a 22-month-old child in Arizona: first report in the United States and a review of the literature. Am J Trop Med Hyg. 2013;88(3):601-5. http://dx.doi.org/10.4269/ajtmh.12-0733 PMID:23382171

10. Otranto D, Giannelli A, Scotty Trumble N, Chavkin M, Kennard G, Latrofa MS, et al. Clinical case presentation and a review of the literature of canine onchocercosis by Onchocerca lupi in the United States. Parasit Vectors. 2015;8(1):89. http://dx.doi. org/10.1186/s13071-015-0699-3 PMID:25884672

11. Otranto D, Dantas-Torres F, Papadopoulos E, Petrić D, Ćupina Al, Bain O. Tracking the vector of Onchocerca lupi in a rural area of Greece. Emerg Infect Dis. 2012;18(7):1196-200. http:// dx.doi.org/10.3201/eid1807.AD1807 PMID:22709878

12. Hermosilla C, Hetzel U, Bausch M, Grübl J, Bauer C. First autochthonous case of canine ocular onchocercosis in Germany. Vet Rec. 2005;156(14):450-2. http://dx.doi. org/10.1136/vr.156.14.450 PMID:15828728

13. Tappe D, Plauth M, Bauer T, Muntau B, Dießel L, Tannich E, et al. A case of autochthonous human Dirofilaria infection, Germany, March 2014. Euro Surveill. 2014;19(17):2-4. http://dx.doi.org/10.2807/1560-7917.ES2014.19.17.20790 PMID:24821120 